Horizons philosophiques

\title{
La génération 68 en France et le changement des valeurs
}

\section{Jean-Marie Apostolidès}

Volume 12, numéro 2, printemps 2002

Valeurs et modernité

URI : https://id.erudit.org/iderudit/801210ar

DOI : https://doi.org/10.7202/801210ar

Aller au sommaire du numéro

Éditeur(s)

Collège Édouard-Montpetit

ISSN

1181-9227 (imprimé)

1920-2954 (numérique)

Découvrir la revue

Citer cet article

Apostolidès, J.-M. (2002). La génération 68 en France et le changement des valeurs. Horizons philosophiques, 12(2), 87-100.

https://doi.org/10.7202/801210ar d'utilisation que vous pouvez consulter en ligne.

https://apropos.erudit.org/fr/usagers/politique-dutilisation/ 


\section{LA GÉNÉRATION 68 EN FRANCE ET LE CHANGEMENT DES VALEURS}

Le soulèvement de Mai 68 en France peut être analysé comme une révolte contre la figure du Père, comme un refus d'héritage de la culture de l'héroïsme'. Les valeurs anciennes y furent critiquées, déconsidérées; on tenta de les remplacer par d'autres, mieux adaptées à la société contemporaine. Certes, les acteurs de Mai n'eurent pas tous des objectifs identiques; si quelques radicaux désiraient une révolution; d'autres, plus modérés - ou plus timorés - ne souhaitaient qu'un aménagement du possible en refusant l'utopie révolutionnaire. Pour ceux-ci, il s'agissait moins de renverser le système social que de l'aménager. Ce sont eux qui ont gagné. C'est dire que si la révolte de Mai n'a pas modifié les fondements économiques et politiques de la société industrielle, elle a eu néanmoins une grande influence sur la mutation des valeurs qui s'en est suivie. Elle a, entre autres choses, permis de passer d'un système social hiérarchisé et patriarcal à un autre, qu'on peut définir comme «fraternel». La structure nouvelle mise en place dans les années qui ont suivi les révoltes de 68 ne signifie certes pas la fin de tout pouvoir mais une nouvelle forme de pouvoir, entraînant dans son sillage de nouveaux comportements et des relations nouvelles entre les êtres. Ce sont les conséquences à long terme de cette mutation des valeurs que nous envisageons ici.

\section{La victoire des vaincus}

Les excès que comporta la révolte de Mai 68 en France amenèrent rapidement un retour de bâton, à savoir une chambre entièrement dominée par la droite aux élections de juin 68. Certains ont pu craindre une remise en selle de l'ordre gaulliste et de la culture de l'héroïsme qui l'accompagnait. Mais cette victoire, loin de marquer le retour des valeurs traditionnelles, en signifie plutôt le déclin. Incapables de faire face aux problèmes apparus lors de la contesta-

1. Par culture de l'héroïsme, nous entendons une tradition dont les éléments remontent autant à l'héritage aristocratique de l'Ancien Régime qu'à la Révolution française (les soldats de l'an II). Cette culture, revivifiée au moment de la Première Guerre mondiale, a pesé d'autant plus lourd au moment de la défaite de 1940 que les Français se sont sentis incapables de la mettre en pratique. Entrée en déclin au moment de la Seconde Guerre mondiale, la culture de l'héroïsme va disparaître avec les guerres coloniales. Elle sera définitivement enterrée par les révoltés de 68 , qui la remplaceront graduellement par une culture de la victimisation. 
tion, et encore moins de leur apporter une solution, les défenseurs de la tradition française vont peu à peu abandonner le terrain idéologique à la génération nouvelle, celle du baby boom. C'est comme s'ils avaient secrètement entériné la castration symbolique à laquelle les événements de 68 les avaient réduits, ce que proclamait fièrement une inscription de la Sorbonne : $" O$ gentils messieurs de la politique vous abritez derrière vos regards vitreux un monde en voie de destruction. Criez, criez, on ne saura jamais assez que vous avez été castrés ${ }^{2}$. Après le départ du Général De Gaulle en 1969, les valeurs nouvelles, proclamées et sacralisées pendant la révolte, vont graduellement se répandre à tous les échelons de la société.

Certes, la transition idéologique ne s'est pas faite en une fois, ni sans accroc. Le gouvernement du Président Pompidou, hanté par le spectre des révoltes, annonciatrices selon lui de révolutions, se trouve soumis à la pression de la partie la plus conservatrice de son électorat. Idéologiquement, il marque un net recul. On voit ainsi pendant ces années d'interrègne un président de formation libérale se replier frileusement sur des valeurs désuètes, par crainte des nouveautés souhaitées par la jeunesse de son pays. L'élection de Valéry Giscard d'Estaing en 1974 saura rattraper le temps perdu et, sans rien changer de fondamental à la direction des affaires, permettra l'avènement d'un libéralisme social et intellectuel qui a pu passer à l'époque pour le vent discret de la liberté.

Malgré, ou en raison des aspects les plus sinistres de la période pompidolienne, le gauchisme continue à gagner du terrain entre 1969 et 1974. En apparence, il est combattu par le gouvernement. Pendant cette période, tout individu portant cheveux longs et arborant des idées libérales est tenu par les pouvoirs publics pour un «ennemi de l'intérieur". Les cars de CRS campent en permanence dans le Quartier latin; les contrôles d'identité sont fréquents et tatillons, pour peu qu'on soit âgé de moins de trente ans et qu'on ne porte pas l'uniforme réglementaire des jeunes cadres dynamiques. II ne fait pas toujours bon d'être jeune dans ces années-là, sous la férule d'un gouvernement dominé par la trouille, et conscient de n'avoir rien d'autre à proposer qu'un affairisme sordide. A défaut d'autorité, le pompidolisme sait exhiber ses matraques pour tenter de contenir un mouvement de contestation qui ne demande qu'à exploser.

2. "Les murs ont la parole", Journal mural de Mai 68, citations recueillies par Julien Besançon, Paris, Tchou éditeur, 1968. Cf. p.28. 
Cependant, sous cette opposition de surface, un redéploiement des forces en présence se poursuit graduellement. En d'autres termes, l'hostilité affichée du pouvoir politique à l'égard de sa jeunesse, particulièrement celle de sensibilité gauchiste, l'indifférence et le mépris dont il fait preuve, dissimulent la lente intégration de cette même jeunesse dans les rouages du pouvoir. Les jeunes y viennent en foule, non pas comme des individus, mais en tant que groupe, en tant que "génération"3. Pour ceux qui le désirent, non seulement la porte s'ouvre, mais elle s'ouvre grand. L'expansion économique aidant (elle se poursuit jusqu'en 1974), ils parviennent aux commandes dans un laps de temps assez bref. Les anciens révoltés de 68 prennent ainsi le pouvoir, particulièrement dans les domaines qui relèvent de la culture: le monde des arts, du cinéma et du théâtre; le monde de l'édition, celui de la presse quotidienne ou hebdomadaire; la publicité et l'ensemble des médias, et, bien sûr, le milieu de l'enseignement. À de nombreux postes ayant la responsabilité de la culture et de l'éducation, on voit peu à peu arriver d'anciens contestataires qui, au prix de quelques compromis et de renoncements moins douloureux qu'ils ne l'auraient supposé, découvrent les plaisirs secrets du commandement et de la gestion. Et comme ils sont intelligents, qu'ils possèdent une capacité d'analyse affinée par quelques années de critique théorique, qu'ils comprennent parfaitement, pour l'avoir contesté quelque temps auparavant, le fonctionnement des rouages de l'État, ils atteignent peu à peu les postes de direction, ce qui leur permet d'exercer une influence certaine sur le devenir idéologique du pays. Ceux de leurs camarades laissés pour compte ne manqueront pas de le leur reprocher.

En d'autres mots, si une partie de la génération de 68 choisit de demeurer du côté des dépossédés, qu'elle s'établisse en usine ou bien qu'elle opte pour une vie marginale en Lozère, une autre partie pense à s'emparer du commandement, surtout dans le domaine qui lui est le plus accessible, celui de la production culturelle et idéologique, où elle fera bientôt régner sa loi. Ces derniers individus deviennent ce que Gramsci a nommé des intellectuels organiques de l'État. Tout en maintenant leur style de vie et certaines de leurs valeurs, ils adaptent les idéaux de la contestation à la situation nouvelle qui leur est faite.

3. Sur la notion de génération, voir Pierre Nora, "La génération", in Les lieux de mémoire, $2^{\circledR}$ édition, Paris, Gallimard, collection Quarto, 1997, pp. 2975-3015. 
Quand nous parlons de prise de pouvoir de la génération de 68 , il ne s'agit pas seulement de la question du renouvellement des élites culturelles. Ce ne sont pas uniquement quelques intellectuels distingués qui donnent désormais le ton à la vie française, c'est tout un groupe, nombreux, qui pèse sur les destinées politiques et économiques du pays. En 68, la classe entière des baby-boomers arrive à l'âge adulte; c'est donc en tant que groupe d'âge, en tant que collectivité qu'elle commence à compter. Elle représente un marché potentiel énorme, elle possède un pouvoir d'achat qui n'est pas négligeable; c'est donc en fonction de ses goûts, de ses valeurs, que les produits de culture seront créés après 68 . La société de consommation fera tout pour flatter cette classe d'âge, pour satisfaire ses goûts, pour aller à la rencontre de ses désirs.

On voit ainsi, à partir du début des années 70 , les valeurs de la révolte prendre une importance toujours plus grande dans les milieux artistiques, intellectuels ou universitaires, au point qu'il n'est pas exagéré de dire que les vaincus de 68 sont en réalité les véritables vainqueurs de cette bataille symbolique. Dynamiques, entreprenants, enthousiastes, porteurs d'un message de bonheur, désireux, après quelque temps d'hésitation, de mettre à la portée de tous les bienfaits de la consommation, ils se font les pourvoyeurs d'une idéologie démocratique nouvelle qu'ils appliqueront partout où ils pourront étendre le réseau de leurs relations ${ }^{4}$. Leur arrivée sur le marché culturel va donner aux années soixante-dix un développement sans précédent. Rétrospectivement, on a l'impression d'un dynamisme dans tous les domaines de la création, des sciences sociales à l'art, en passant par le cinéma, la musique pop ou la philosophie. Les sciences humaines, la linguistique et l'histoire connaissent aussi une floraison sans précédent. Tous les domaines du réel sont explorés, jaugés, analysés dans leurs dimensions cachées. II n'est plus possible d'avoir une interprétation simple des événements ou du langage; dans le sillage des philosophies du XIXe siècle, les structures profondes du réel sont mises à jour, la ruse de la raison dévoilée, les illusions de l'idéologie mises à nu par ses célibataires mêmes. Les éditeurs, encouragés par un lectorat avide de découvertes, font preuve d'une témérité qui n'aura qu'un temps. Tout ce qui porte le sceau de la nouveauté est accueilli avec un mélange de générosité et d'esprit critique qui témoigne d'une ébullition intellectuelle intense. La position de la

4. François Ricard a bien analysé les caractéristiques de cette génération dans un livre qui a fait date, La génération lyrique. Essai sur la vie et l'œuvre des premiersnés du baby-boom, Montréal, Editions du Boréal, 1992. 
génération de 68 est idéale puisque si d'un côté elle détient le pouvoir culturel effectif, de l'autre elle promeut un mode de pensée et des valeurs qui paraissent encore s'opposer aux valeurs dominantes et qui constituent pour le public autant de découvertes. Dans un tel climat, la moindre nouveauté fait figure de conquête; on a le sentiment d'explorer des continents obscurs ou mal connus, sous la bannière de mentors jeunes, audacieux, qui ont su échapper aux ornières de la tradition. Les intellectuels en vogue engrangent alors tous les bénéfices, ceux de la jeunesse, ceux du pouvoir et ceux de la contestation.

Le programme culturel de la génération du baby boom peut se résumer d'un mot, la libération. Pour cette génération, il est l'équivalent de ce qu'était le terme "lumière" pour les philosophes du XVIIIle siècle. En 1970, il ne s'agit plus seulement d'éclairer, il faut libérer de leurs contraintes tous ceux qui paraissent enchaînés à la culture ancienne de l'héroïsme, désormais déconsidérée dans la plupart de ses aspects. On veut libérer les peuples opprimés, les femmes, les homosexuels, les malades mentaux, les handicapés, c'est-à-dire toute personne qui ne jouit pas d'un accès libre et total au bien-être matériel. Sous la bannière de Marx, de Nietzsche et de Freud, on met à jour les aliénations héritées de la société bourgeoise. Si la libération est le mot passe-partout, l'égalité est la valeur suprême. La sensibilité aux injustices, aux hiérarchies, se fait plus exacerbée; on considère d'un œil nouveau les rapports hommes-femmes, patrons-employés, maîtres-élèves, et même parents-enfants. Tout ce qui porte l'estampille de la hiérarchie est tenu en suspicion. L'égalité et la démocratie se répandent dans tous les milieux, s'installent entre tous les êtres. On se porte au secours de ceux qui n'y ont pas le même accès. On dénonce partout les ruses du pouvoir, et la collaboration implicite de toute personne qui accepte de s'y soumettre. On rêve, comme au plus fort de la contestation de Mai 68 , d'une vie supérieure accessible à tous, une vie de maîtres sans esclaves, pour reprendre l'expression de Raoul Vaneigem 5 . Et ce déferlement de bons sentiments paraît d'autant plus généreux qu'il provient de gens qui se sentent en sympathie profonde avec les opprimés, qui se vivent eux-mêmes parfois comme des victimes. En bref, on espère fonder cette communauté utopique qui est le rêve secret des baby boomers.

5. Voir le Traité de savoir-vivre à l'usage des jeunes générations, qui date de 1967. 


\section{Le style copain}

La victoire des révoltés de 68 entraîne une modification des rapports humains dont l'abolition apparente des hiérarchies est la marque la plus visible. Elle est la conséquence de la critique de l'autorité et du système patriarcal qui a été menée pendant les années soixante et s'est poursuivie pendant la décennie suivante. Elle est l'expression la plus visible du mythe communautaire qui caractérise, selon nous, la génération du baby boom. Étant donné les archaïsmes de la culture traditionnelle et les contraintes que les dysfonctionnements des valeurs anciennes faisaient peser sur la majorité de la population, l'écroulement soudain des marques de hiérarchie a été vécu unanimement comme une libération, même par les tenants de l'autorité. Comme s'ils se trouvaient tout d'un coup débarrassés d'un poids insupportable. À la place des relations traditionnelles, figées dans un formalisme désuet, on voit s'installer dans les années 70 un "style copain" qui emprunte autant à la tradition révolutionnaire française qu'à l'Amérique contemporaine. II permettra des relations inter-personnelles plus détendues dans un climat de tension et d'incertitude. Le capital symbolique ancien est mis de côté; les différences hiérarchiques sont oubliées, au bénéfice d'une liberté et d'une égalité plus grandes entre les êtres.

La démocratisation des relations humaines se remarque autant dans le changement de costume (le complet-cravate cessant d'être une obligation quotidienne) que dans les signes de reconnaissance. Le tutoiement se fait plus fréquent; l'utilisation du prénom tend à supplanter les formes anciennes de distinction comme "monsieur", "madame», "docteur», "maître», «monseigneur" ou "mon père», encore en usage jusqu'en 68. L'abolition des marques hiérarchiques entraîne non seulement une plus grande proximité entre les êtres mais aussi la fin de la séparation. En d'autres termes, les différences individuelles s'amenuisent au bénéfice d'un comportement identitaire. Désormais, l'idéal consiste moins à se placer en situation d'extériorité qu'à s'intégrer à un groupe. L'individu ne veut plus se tenir en dehors, dans une position séparée, qui implique une responsabilité individuelle et un jugement. II refuse d'être un individu; il veut devenir comme les autres, semblable à eux, même au prix d'un amoindrissement de sa personnalité. Dans les milieux du travail comme dans l'espace privé, on cherche moins à imposer une vision personnelle qu'à s'intégrer à un groupe. Il s'agit de se mettre d'accord, ensemble, sur des choix, de prendre des décisions en commun, d'aboutir à un 
consensus qui paraît indispensable à la création de la communauté. Inutile d'insister sur le fait que cette démocratisation des mœurs entraîne avec elle son lot de négativité. Si d'un côté elle est une libération des liens traditionnels, devenus inadaptés et inefficaces, d'un autre côté elle peut à l'occasion produire un écrasement des positions individuelles au profit du groupe. La recherche du consensus se fait au détriment des solutions individuelles, parfois plus originales ou plus audacieuses. Le conformisme et la médiocrité peuvent s'imposer chaque fois qu'apparaissent des difficultés, et la tyrannie du groupe risque de devenir aussi pesante qu'étaient autrefois les abus de l'autorité.

L'adoption du style copain définira l'adhésion aux idéaux de 68. Elle engendrera en outre la multiplication et la rapidité des échanges. En se redoublant au niveau de la technologie informatique, elle permettra de transformer l'ensemble du territoire en un immense réseau d'échanges et de communications qui n'a cessé de se complexifier depuis lors. Le style copain constituera aussi un signe de reconnaissance entre gens partageant les mêmes valeurs. À la place des symboles de la hiérarchie, on verra les signes de l'identité s'installer entre les êtres. Et ceux-ci feront image, se transformeront en images qui constitueront les nouvelles médiations entre les hommes.

Ajoutons enfin que le style copain se dégrade à l'occasion en simple "copinage». Dans le domaine de la culture, les vainqueurs de 68, une fois parvenus au pouvoir, auront tendance à fermer la porte derrière eux, à n'élire que les œuvres de leurs pairs pour en faire la promotion. On se cite, on s'encense mutuellement, on s'admire dans les productions des gens d'une même promotion. Peu à peu, ce petit jeu de pouvoir entraîne un appauvrissement général. Après l'explosion intellectuelle et artistique des années soixante et soixante-dix, si l'on assiste en France à un essoufflement dans le domaine des idées, c'est peut-être en partie dû au fait que le copinage institué crée non seulement une censure sournoise sur laquelle viennent achopper les idées véritablement nouvelles mais aussi un conformisme dans le milieu intellectuel qui se perpétue aujourd'hui. L'émergence d'un nouveau type d'intellectuel, choyé par les médias, ajoute encore à la confusion, permettant de confondre visibilité intellectuelle et originalité. Loin de susciter un nouveau débat, le mariage entre le milieu intellectuel et la télévision a surtout engendré une production du même : on reprend ce qui est déjà connu; on répète les mêmes 
idées, les mêmes formules, comme si les créations intellectuelles et artistiques possédaient le même statut que les marchandises industrielles et devaient se modeler les unes sur les autres pour devenir consommables par le grand public.

\section{La civilisation des frères}

Nous constatons ainsi que la principale conséquence de la révolte de 68 a été la mise à mort du système symbolique lié à la culture ancienne, à savoir le patriarcat. Toutes les valeurs, tous les éléments liés à la figure du Père - clé de voûte du système - se trouvent critiqués les uns après les autres, autant dans le domaine politique que dans celui des relations sociales. La famille nucléaire, soudée autour de la figure paternelle, se mue en une institution fragile, provisoire, incertaine. Entre 1972 et 1990, le nombre des divorces en France passe de 43000 à 106000 . Un couple sur trois se trouve touché, et un sur deux en région parisienne. Selon certaines estimations, les "enfants du divorce» avoisinent 1200000 en 1994, soit $9 \%$ des mineurs. Après le divorce, le remariage d'un conjoint (dans $18 \%$ des cas), ou une nouvelle union dite libre, vient compliquer la situation. Dans le même temps, les familles monoparentales (la mère, dans $90 \%$ des cas) se multiplient : plus d'un million en 1994, contre 660000 en 1968)6. Des valeurs qui paraissent liées au patriarcat, comme la responsabilité ou l'universalité, sont critiquées. Elles sont abandonnées au nom du droit à la différence. Bref, tout ce qui portait auparavant l'estampille du Père se trouve frappé de suspicion, sinon même arraché, comme les Révolutionnaires de 93 tentèrent d'effacer des édifices publics les marques de l'Ancien Régime. À la place d'un système symbolique plaçant les êtres en hiérarchie les uns par rapport aux autres, on voit s'établir un système de signes, fondé sur l'égalité abstraite de tous les individus, et qui ne prend en compte que les différences quantifiables, à savoir leur degré de fortune.

Élevés dans la culture de l'héroïsme au moment de son déclin, les enfants du baby-boom en ont refusé l'héritage, ou plutôt ils ont refusé de le transmettre à la génération qui les a suivis. Cette dernière ne recevra généralement ni culture politique ni culture religieuse. Un sondage réalisé par le CREDOC en 1993, et dont le thème était les valeurs à transmettre aux enfants, témoigne de ce refus de la transmission. Une majorité de parents $(53 \%)$ estiment qu'il ne leur

6. J'emprunte ces données à Michel Fize, Le peuple adolescent, Paris, Julliard, 1994, pp. 36-37. 
appartient pas «d'inciter leurs enfants à s'intéresser aux grands choix politiques". Même attitude dans le domaine religieux. Sous prétexte de ne pas s'imposer de manière autoritaire à leur progéniture, $52,6 \%$ des parents pensent qu'il n'est pas (ou peu) important que leurs enfants découvrent la foi en Dieu. Plus inquiétant, $54,2 \%$ ne jugent pas important qu'ils s'initient à une culture religieuse. Ce sont des sujets dont on ne parle pas en famille ${ }^{7}$. On constate une même démission dans le milieu de l'enseignement, particulièrement en ce qui a trait à la culture humaniste. On remarque chez de nombreux enseignants français un refus implicite de transmettre l'héritage culturel qui fut le leur. Sous prétexte qu'ils ne souhaitaient pas enseigner des valeurs désuètes ou déconsidérées, les baby-boomers, parvenus aux commandes après 68 , ont éliminé de l'enseignement un savoir traditionnel, constitué par de nombreuses générations, et qui servait de lien et de langage commun entre elles. Loin de demeurer un lieu abstrait, coupé du quotidien, dans lequel l'élève pouvait faire une économie d'expérience en ayant accès à des valeurs, des comportements et des problèmes qu'il n'avait jamais rencontrés auparavant, l'école s'est transformée peu à peu en un lieu redoublant l'espace social extérieur, dans lequel on lui demandait de redire, de retrouver le monde du dehors qui lui était familier. Sous couvert de se mettre à sa portée, les maîtres l'enfonçaient davantage dans un réalisme auquel il ne pouvait plus échapper, même par l'imagination. Toute tradition qui permettait une perspective critique sur le temps présent, a été ainsi déconsidérée au nom d'une approche concrète des problèmes. Le résultat le plus visible a été de priver la jeune génération des instruments de la critique, instruments qui avaient permis à leurs parents de réaliser leur prise de pouvoir en 68 .

Au risque de tomber dans le schématisme, essayons de comprendre certains phénomènes qui se sont déroulés depuis un quart de siècle. En 1968, les enfants du baby-boom se révoltent contre la génération de leurs parents, généralement déconsidérée en raison de son attitude peu glorieuse durant la Seconde Guerre mondiale. Ils se révoltent, mais d'un autre côté ils accomplissent le rêve secret de leur parents, à savoir cet acte héroïque de révolte que la génération précédente n'avait pu, ou n'avait osé faire lorsqu'elle s'était placée presque tout entière sous la houlette du Maréchal Pétain. En 68, les baby-boomers ne se contentent pas de critiquer leurs aînés et de prendre leur place, ils mettent à bas l'héritage

7. Ibid., p. 50 . 
spirituel et intellectuel qu'ils ont reçu pour lui préférer des valeurs nouvelles, dont ils sont partiellement les inventeurs, et dont nous avons dit qu'elles visaient à établir une communauté. En triomphant aisément de leurs parents au cours d'un sociodrame collectif ayant ébranlé la société française jusque dans ses fondements, ils refusent du même coup la place symbolique qui aurait dû être la leur après cette victoire, à savoir la place du Père dans la structure traditionnelle. Ils s'emparent des commandes moins comme des héros que comme membres d'une génération, d'une communauté soudés par la même expérience. Ils prennent le pouvoir, certes, mais cette prise de pouvoir ne se transforme pas en victoire car elle n'est pas symbolisée comme un acte héroïque. Ils restent ainsi des vainqueurs honteux, des hommes de pouvoir qui n'assument pas vraiment leur position. En d'autres termes, faute de s'intégrer dans l'ensemble symbolique de la culture héroïque, ils vont se contenter au cours des années suivantes, de célébrer des anniversaires, à commencer par celui de 68 , afin de se convaincre eux-mêmes, et de convaincre tout le monde autour d'eux, qu'il s'est bel et bien passé quelque chose, que leur conquête est légitime, et qu'elle participe de la mémoire collective. Faute d'accéder au symbolisme, ils auront le pouvoir, mais s'arrangeront toujours pour le nier, pour ne pas l'assumer clairement, surtout vis-àvis de la génération qui les suit.

Mêmes conséquences au niveau de l'éducation. Parce que les baby-boomers refusent le patriarcat, ils ne désirent pas devenir des pères, des modèles d'autorité, des incarnations de la Loi, comme cela s'était toujours produit auparavant; ils veulent des enfants qui leur ressemblent, du moins l'affirment-ils. Sur les murs de la Sorbonne en 68 , on relève cette inscription exemplaire : «Ne faites pas votre testament avant de mourir pour un idéal, faites un gosse qui soit digne de

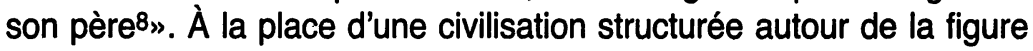
paternelle "héroïque», ils établissent une civilisation des frères, dont nous pouvons entrevoir certaines caractéristiques : abaissement des différences entre le masculin et le féminin; promotion de l'enfant au statut imaginaire de centre de la vie affective; critique de toutes les valeurs liées au patriarcat et à l'héroïsme; invention d'un star system auquel tout le monde doit participer. La civilisation des frères se fonde sur une plus grande égalité entre les participants, particulièrement entre hommes et femmes ${ }^{9}$; elle vise à prévenir l'émergence de toute

8. Les murs ont la parole, p. 28.

9. Cf. Carole Pateman, The Sexual Contract, Stanford, Stanford University Press, 1988. Voir aussi Juliet MacCannell, The Regime of the Brother : After the Patriarchy, London, Routledge, 1991. 
figure susceptible d'en menacer l'équilibre, surtout les figures autoritaires. À la place des symboles de l'autorité, elle préfère les images qui établissent des médiations nouvelles entre les êtres et les choses. Cependant, une fois aux postes de commande, les frères ne parviennent pas à s'unir. Ils sont constamment en rivalité les uns avec les autres. Ils se font concurrence, faute de s'accorder sur un ordre symbolique.

\section{La génération candide}

Lorsque la génération du baby-boom atteint l'âge d'homme et devient à son tour capable d'éduquer ses propres enfants, c'est-à-dire après la victoire de 68 , elle s'attelle à cette tâche en fonction des valeurs qui lui sont propres. Non seulement refuse-t-elle de transmettre le savoir qu'elle a reçu en héritage, sous prétexte qu'il est entaché de valeurs qu'elle désapprouve, mais encore refuse-t-elle d'assumer à l'égard de sa progéniture la fonction paternelle. En d'autres termes, elle ne veut pas créer de conflit avec ses fils; elle ne souhaite ni séparation ni hiérarchie. Elle n'a guère envie de rencontrer l'opposition qui est le lot de toute figure autoritaire s'assumant comme telle. À la place du Père autoritaire de la culture héroïque, qui dit non et se fait au moment de l'adolescence des enfants l'incarnation de la Loi, elle promeut le père copain, celui qui sait rester jeune et qui, loin d'entrer en conflit avec son fils, le laisse croître à son propre rythme, sans lui imposer de contrainte ni lui faire violence. Davantage un frère aîné qu'un père "sévère", le père d'après soixante-huit se met à l'écoute de son enfant. Toujours prêt à négocier avec lui, il cherche d'autant moins à imposer ses solutions qu'il est incertain de ses propres valeurs. Se refusant à faire de la discipline, il préférera, outre les vertus du dialogue, l'humour et la dérision qui sont les meilleurs dissolvants de l'autorité.

Élevés dans une ambiance débordante d'affection, les enfants des baby-boomers, ceux nés à partir de 1970, ont d'autant moins cherché à se séparer de leurs parents qu'ils ont affronté une crise économique qui, jusqu'à aujourd'hui, a rendu difficile la recherche d'un travail pour la majorité d'entre eux. Mais, parce qu'ils n'ont jamais été confrontés à des figures autoritaires leur indiquant clairement la voie à suivre, ces enfants ont été privés de la possibilité même de révolte. On ne saurait se révolter contre un grand frère qui, tout en vous faisant part de ses propres doutes, vous protège ou du moins 
vous assure le minimum. Comment s'opposer à la tyrannie de la douceur et de la compréhension? À la rigueur, on préfère l'indifférence à l'hostilité ouverte. Comment quitter la maison alors que l'inquiétante perspective du chômage rend le monde extérieur plus angoissant qu'un univers familial instable et sans règlements clairement définis?

Les conséquences de cet état de fait me paraissent les suivantes. D'une part, la génération du baby-boom a fermé la porte derrière elle dès qu'elle s'est elle-même sentie menacée par le chômage, après la crise économique de 1974. Après avoir pris les places à la suite du coup de force de 68 , elle a tenté de se maintenir au sommet à un moment où l'arrivée de nouvelles technologies et l'avènement de la mondialisation des échanges rendaient la situation de l'emploi précaire dans tous les pays d'Europe de l'ouest. Elle a plus ou moins consciemment refusé de transmettre son savoir-faire à ses propres enfants, non seulement parce qu'elle ne croyait plus aux valeurs de la culture héroïque, mais encore parce qu'elle ne souhaitait pas voir se dresser devant elle des rivaux potentiels qui auraient pu la déloger de ses emplois le moment venu. Le mimétisme, la concurrence, la rivalité pour le pouvoir sont des caractéristiques de la civilisation des frères. Pour éviter une telle situation, la génération de 68 a préféré sacrifier la génération de ses fils. Et elle l'a fait de façon sournoise, honteuse, sans le dire franchement, en maintenant sa progéniture dans un état de tutelle permanente, en l'étouffant sous les bons sentiments, la tendresse et la compréhension. En la forçant surtout à s'intéresser aux victimes à un point tel qu'il s'est créé une identité inconsciente entre eux et les damnés de la terre. Écrasés par la génération précédente qui leur paraissait d'autant plus forte qu'elle avait refusé de divulguer le secret de sa victoire, anxieux parce qu'éduqués dans un climat d'incertitude où ils ne se confrontaient jamais à l'autorité, les enfants des baby-boomers sont généralement demeurés dans l'ombre de leurs parents. Ils ont souvent opté pour deux attitudes opposées, soit la répétition parodique des valeurs de leurs parents, soit l'indifférence à leur égard et la constitution d'une culture séparée, ne permettant aucun échange avec la génération des aînés. Dans tous les cas, ils ont endossé le masque d'innocence que leurs parents avaient préparé, ce qui nous incite à proposer pour eux l'appellation de "génération candide". Comme le personnage du conte de Voltaire qui, une fois loin du château du baron de Thunder-ten-tronckh, se trouve confronté à un univers terrible, dominé par la cruauté et 
l'échange marchand, et dans lequel il ne lui arrive que des malheurs qu'il subit passivement en attendant de réaliser son rêve le plus cher, à savoir reconstituer autour de lui l'atmosphère sécurisante du monde de son enfance, les rejetons des baby-boomers sont innocents du malheur qu'ils perçoivent autour d'eux. Même s'ils ne croient pas que tout est pour le mieux dans le meilleur des mondes possibles, ils souhaitent améliorer leur environnement en refusant la violence, l'exploitation, l'injustice. En même temps, ils sympathisent avec toutes les victimes. Ils désirent que tous les gens aient un même accès au bonheur matériel; ils veulent la fin de la discrimination, du racisme, de la violence, sans pour autant renoncer aux avantages du système économique qui les a produits.

Ainsi, pour des raisons à la fois culturelles et économiques, c'est le mécanisme même de la succession des générations qui s'est trouvé faussé après 68 . En refusant d'assumer le rôle du Père qu'ils avaient vaincu au moment de la révolte, les baby-boomers ne se sont pas seulement assuré une victoire honteuse, qui leur a donné le pouvoir à défaut de leur valoir l'autorité; ils ont également coupé l'herbe sous le pied de la génération candide, l'empêchant de se révolter, c'est-à-dire lui interdisant implicitement de mûrir et de prendre sa place. La ruse de la culture fraternelle consiste à maintenir les jeunes dans une dépendance perpétuelle, faite de crainte et de soumission, à les forcer à endosser les valeurs de leurs aînés, sans les critiquer, sans pouvoir les rejeter autrement que par des marques d'indifférence. D'où le ressassement des modes culturelles rétro et des valeurs des années soixante auquel on assiste depuis vingt ans et l'interdiction tacite d'autres formes artistiques ou d'autres modes intellectuelles en rupture avec les valeurs du gauchisme soixantehuitard. Faute de s'être révoltée, la génération candide est demeurée dans l'ombre du Big Brother protecteur et intimidant. Et si ce dernier est resté en position de force jusqu'à aujourd'hui, c'est parce qu'il a refusé de prendre place dans un système symbolique qui, en instituant une structure dans laquelle tout un chacun trouverait son rôle, aurait permis la transmission d'une généalogie. En d'autres mots, la destitution du patriarcat et de l'héroïsme en 68 a engendré un procès de dé-symbolisation dans lequel la génération candide s'est trouvé piégée. Faute de rencontrer une figure autoritaire et des normes auxquelles s'opposer, elle s'est éloignée dans l'indifférence ou bien s'est tenue dans le sillage de ses aînés, faisant preuve à leur égard d'une docilité qui a prévenu toute révolte. Ceux qui ont tenté un autre 
chemin ont été écrasés sous la critique ou bien sous le silence, stratégie habituelle des gens en place qui ne désirent pas que de nouvelles voix se fassent entendre.

\author{
Jean-Marie Apostolidès
} Université de Stanford 